\title{
Potential Association of Body Constitution with the Prognosis of IgA Nephropathy: A Long-Time Follow-Up of 203 Cases in China
}

\author{
Linchang Liu $\left(\mathbb{D},{ }^{1,2}\right.$ Zhiwei Yin ${ }^{(D)}{ }^{1}$ Jiwei Ma ${ }^{D},{ }^{1}$ Shuwei Duan ${ }^{(D)},{ }^{1}$ and Xiangmei Chen ${ }^{1}$ \\ ${ }^{1}$ Department of Nephrology, Chinese People's Liberation Army General Hospital (301 Hospital), \\ Chinese People's Liberation Army Institute of Nephrology, State Key Laboratory of Kidney Diseases, \\ National Clinical Research Center for Kidney Diseases, Beijing Key Laboratory of Kidney Diseases, Beijing 100853, China \\ ${ }^{2}$ Department of Rheumatology and Nephrology, Zhejiang Integrated Traditional and Western Medicine Hospital, \\ Hangzhou 310003, China \\ Correspondence should be addressed to Xiangmei Chen; xmchen301@126.com
}

Received 26 February 2019; Revised 25 April 2019; Accepted 3 June 2019; Published 23 June 2019

Academic Editor: Morry Silberstein

Copyright (C) 2019 Linchang Liu et al. This is an open access article distributed under the Creative Commons Attribution License, which permits unrestricted use, distribution, and reproduction in any medium, provided the original work is properly cited.

\begin{abstract}
Objective. This study investigated the association between body constitution (BC) and the prognosis of IgA nephropathy. Methods. We analyzed 203 biopsy-diagnosed IgA nephropathy patients, who were followed up for (63.9 \pm 16.2$)$ months. The participants' BC statuses were evaluated with the Constitution in Chinese Medicine Questionnaire; the relationships between clinical parameters and renal outcomes were analyzed by Cox regression. Results. Patients were classified into chronic kidney disease stages with $43.4 \%$ in stage $1,27.1 \%$ in stage $2,26.1 \%$ in stage 3, 3.5\% in stage 4 , and none in stage 5. Qi-deficiency BC type was the most common BC type in IgA nephropathy patients. In univariate analysis, proteinuria of more than $\mathrm{lg} / \mathrm{d}$, hypertension, renal impairment (estimated glomerular filtration rate $<60 \mathrm{~mL} / \mathrm{min}$ ), hypoproteinemia, hyperuricemia, Yang-deficiency BC, and blood-stasis BC were associated with poor prognosis. Multivariate analysis identified that hypertension (hazard ratios (HR) 3.5, $P=0.009$ ), renal impairment (HR 5.8, $P<0.001$ ), Yang-deficiency BC (HR 2.3, $P=0.041$ ), and blood-stasis BC (HR 2.5, $P=0.017$ ) were independent predictors of unfavorable renal outcomes. Conclusions. Most patients of IgA nephropathy were biopsied at an early stage. Yang-deficiency BC and bloodstasis $\mathrm{BC}$ at biopsy were most closely associated with the worse prognosis of IgA nephropathy along with hypertension and renal impairment.
\end{abstract}

\section{Introduction}

Body constitution (BC), a distinct characteristic of an individual in Traditional Chinese Medicine (TCM), can affect the progression of diseases $[1,2]$. Yang-deficient $\mathrm{BC}$ is associated with the prognosis of diabetic nephropathy [3], Yang-deficient BC can be identified as a potential predictor of early HIV-related mortality, and side effects in patients initiated highly active antiretroviral therapy [4]. Blood-stasis $B C$ is associated with peripheral arterial disease in patients with type 2 diabetes [5]. However, the role of $\mathrm{BC}$ in the longterm prognosis of IgA nephropathy is unclear.

IgA nephropathy is the most common primary glomerulonephritis worldwide [6]. The clinical course of IgA nephropathy is highly variable, ranging from asymptomatic microscopic hematuria to end-stage renal disease (ESRD)
[7]. Previous studies have described some risk factors in the prognosis of IgA nephropathy $[8,9]$. We investigated the association between BC and the prognosis of IgA nephropathy.

\section{Materials and Methods}

2.1. Subjects. From May 2010 to October 2014, a total of 350 patients newly diagnosed for IgA nephropathy were enrolled in this study within three days before and after renal biopsy in the Department of Nephrology, Chinese People's Liberation Army (PLA), General Hospital, and 203 cases were followed for more than 48 months. These 203 cases composed the study group, who came from seven provinces or municipalities of Northern China (Neimenggu, Hebei, Henan, Shanxi, Shandong, Beijing, and Tianjin). The 
inclusion criteria were as follows: (i) biopsy-proven primary IgA nephropathy; and (ii) age $>14$ years. The exclusion criteria were as follows: (i) IgA nephropathy secondary to chronic liver disease, henoch-schonlein purpura, systemic lupus erythematosus, and other autoimmune disorders; (ii) accompanied with acute interstitial nephritis or diabetes mellitus; and (iii) TCM therapy within the past 2 months. From May 2010 to December 2010, 64 subjects of Chinese college students and community residents without chronic disease in Beijing city were enrolled as the control group. All of the research procedures were approved by the Ethics Committee of the General Hospital of the Chinese PLA, and all subjects had signed an informed consent.

2.2. Clinical Information. The baseline data at the time of renal biopsy were collected. This information included sex, age, lifestyle behaviors (smoking, alcohol drinking, and less exercise), body mass index (BMI), presence of hypertension (blood pressure $>140 / 90 \mathrm{mmHg}$ or requirement for antihypertensive therapy), $24 \mathrm{~h}$ urinary protein excretion, serum albumin, creatinine, and uric acid levels, corticosteroid and/or immunosuppressant treatment. Serum albumin, creatinine, and uric acid levels were measured by a Roche MODULAR automatic biochemistry analyzer. Mean arterial pressure (MAP) was defined as diastolic pressure plus a third of the pulse pressure. The estimated glomerular filtration rate (eGFR) was calculated by the Chronic Kidney Disease Epidemiology Collaboration (CKD-EPI) equation adjusted for Asian populations [10]. ESRD was defined as eGFR $<15 \mathrm{ml} / \mathrm{min} / 1.73 \mathrm{~m}^{2}$, initiation of dialysis, or kidney transplantation. Renal endpoint (combined event) contained $50 \%$ reduction in eGFR or ESRD. Follow-up data (updated to June 2018) were collected via outpatient and telephone interview by two independent clinical reviewers.

2.3. Renal Biopsy. The renal biopsies were performed by the director of Department of Nephrology in our hospital. The biopsy specimens were processed for immunofluorescence, light microscopy, and electron microscopy studies. The diagnostic criteria of IgA nephropathy were as follows: (i) IgA deposited in mesangial for at least $1+$ (on a scale from 0 to $4+$ ) in immunofluorescence studies, and the dominant immunoglobulin deposited in the glomeruli was IgA, and (ii) electron-dense deposited in mesangial in EM studies [11].

2.4. BC Identification. The Constitution in Chinese Medicine Questionnaire (CCMQ), issued by the China association of TCM in 2009, was adopted to evaluate the participants' BC statuses [4].

All of the participants were asked to complete the CCMQ, which consists of 60 items rated on a 5-point Likert Scale. The scores of all items in each subscale were summarized; then, the sum scores were converted for identifying the participants' BC types. The CCMQ is grouped into nine $\mathrm{BC}$ types: gentleness BC, Yang-deficiency BC, Yin-deficiency BC, Qi-deficiency BC, phlegm-dampness BC, dampness-heat BC, blood-stasis BC, Qi-depression BC, and special diathesis BC types. Gentleness $\mathrm{BC}$ type is the only balanced $\mathrm{BC}$ type, and the other eight $\mathrm{BC}$ types are unbalanced $\mathrm{BC}$ types.
2.5. Statistical Analysis. Patients were stratified by their BC types. Continuous variables were presented as the mean \pm standard deviation (SD); categorical variables were presented as a number and percentage. The differences between two groups were compared by the unpaired t-test and $\chi^{2}$ test, as appropriate. The Kaplan-Meier method was used to estimate the renal survival. The relationships between factors and renal survival were assessed with Cox regression. Factors showing statistical significance in univariate analysis were further considered with multivariate analysis. The significance level was $P<0.05$ (two-sided). All statistical analyses were performed using SPSS 17.0 software (SPSS, Chicago, IL, USA).

\section{Results}

3.1. Demographic and Clinical Features. We investigated 203 IgA nephropathy patients in this study, with an average age of $(40.1 \pm 11.6)$ years, and 111 cases $(54.7 \%)$ were males. $36.9 \%$ of these patients had hypertension. The proteinuria level was $(1.68 \pm 1.57) \mathrm{g} / \mathrm{d}, 53.7 \%$ of our patients had proteinuria greater than $1.0 \mathrm{~g} / \mathrm{d}$, and $11.3 \%$ had proteinuria greater than $3.5 \mathrm{~g} / \mathrm{d}$. Patients were classified into chronic kidney disease (CKD) stages with $43.4 \%$ in stage $1,27.1 \%$ in stage $2,26.1 \%$ in stage $3,3.5 \%$ in stage 4 , and none in stage 5 . The mean follow-up period was $(63.9 \pm 16.2)$ months. During follow-up, 32 patients (15.8\%) achieved the renal endpoint event. There were 64 participants in the control group, with an average age of $(42.4 \pm 18.4)$ years and 34 participants (53.1\%) were males.

In this study, the most common BC type was Qideficiency BC type; the proportion of Qi-deficiency BC type was $56.2 \%$ in the $\operatorname{IgA}$ nephropathy group and $37.5 \%$ in the control group. The gentleness $\mathrm{BC}$ type percentage was lower in the IgA nephropathy group than the control group $(P=0.027)$. The proportions of Qi-deficiency BC, phlegmdampness BC, dampness-heat BC, blood-stasis, and special diathesis $\mathrm{BC}$ types were higher in the $\operatorname{IgA}$ nephropathy group than the control group $(P<0.05)$ (Table 1$)$. In IgA nephropathy group, $98.0 \%$ of cases could be classified for at least one BC type, and $67.5 \%$ had more than one imbalanced BC type. In the control group, the percentage was $96.9 \%$ for at least one BC type and $55.3 \%$ for more than one imbalanced BC type (Table 2).

3.2. Distribution of Yang-Deficiency BC. The patients were divided into Yang-deficiency BC group (97, 47.8\%) and nonYang-deficiency BC group (106, 52.2\%). At baseline, there were no differences between the two groups in clinical characteristics such as age, BMI, lifestyle behaviors (smoking, alcohol drinking, and less exercise), MAP, initial proteinuria, eGFR, serum uric acid, CKD stage, and corticosteroid and/or immunosuppressant treatment. The male percentage was lower in the Yang-deficiency BC group than the nonYang-deficiency BC group $(P=0.011)$. The serum albumin level was lower in the Yang-deficiency $\mathrm{BC}$ group than the non-Yang-deficiency BC group $(P=0.009)$. During followup, more patients achieved the renal endpoint in Yangdeficiency BC group (23 (23.7\%)) than non-Yang-deficiency BC group (9(8.5\%), $P=0.003$, Table 3$)$. Yang-deficiency BC 
TABLE 1: Distribution of BC in IgA nephropathy group and the control group.

\begin{tabular}{lccc}
\hline Characteristics & IgA nephropathy group $(\mathrm{n}=203)$ & the control group $(\mathrm{n}=64)$ & $P$ values \\
\hline Age (years) & $40.1 \pm 11.6$ & $42.4 \pm 18.4$ & 0.352 \\
Male (n (\%)) & $111(54.7)$ & $34(53.1)$ & $18(28.1)$ \\
Gentleness BC (n (\%)) & $32(15.8)$ & $23(35.9)$ & 0.828 \\
Yang-deficiency BC (n (\%)) & $97(47.8)$ & $23(35.9)$ & 0.027 \\
Yin-deficiency BC (n (\%)) & $80(39.4)$ & $24(37.5)$ & 0.619 \\
Qi-deficiency BC (n (\%)) & $114(56.2)$ & $19(29.7)$ & 0.009 \\
Phlegm-dampness BC (n (\%)) & $90(44.3)$ & $16(25.0)$ & 0.038 \\
Dampness-heat BC (n (\%)) & $93(45.8)$ & $12(18.8)$ & 0.003 \\
Blood-stasis BC (n (\%)) & $64(31.5)$ & $10(15.6)$ & 0.048 \\
Qi-depression BC (n (\%)) & $41(20.2)$ & $15(23.4)$ & 0.417 \\
Special diathesis BC (n (\%)) & $80(39.4)$ & $2(3.1)$ \\
No BC (n (\%)) & $4(2.0)$ & 0.020 \\
\hline
\end{tabular}

TABLE 2: Number of BC types in IgA nephropathy group and the control group (n (\%)).

\begin{tabular}{lcc}
\hline Number of BC types & IgA nephropathy group $(\mathrm{n}=203)$ & the control group $(\mathrm{n}=64)$ \\
\hline 0 & $4(2.0)$ & $2(3.1)$ \\
1 & $62(30.5)$ & $26(40.6)$ \\
2 & $22(10.8)$ & $7(10.9)$ \\
3 & $27(13.3)$ & $7(21.9)$ \\
4 & $19(9.4)$ & $2(3.1)$ \\
5 & $25(12.3)$ & $2(3.1)$ \\
6 & $15(7.4)$ & $4(6.3)$ \\
7 & $19(9.4)$ & $0(0)$ \\
8
\end{tabular}

TABLE 3: Clinical characteristics at biopsy and follow-up for patients with Yang-deficiency BC and non-Yang-deficiency BC.

\begin{tabular}{|c|c|c|c|}
\hline Characteristics & Yang-deficiency BC $(\mathrm{n}=97)$ & Non-Yang-deficiency BC $(\mathrm{n}=106)$ & $P$ values \\
\hline Age (years) & $41.0 \pm 10.9$ & $39.2 \pm 12.1$ & 0.271 \\
\hline Male (n (\%)) & $44(45.4)$ & $67(63.2)$ & 0.011 \\
\hline BMI $\left(\mathrm{kg} / \mathrm{m}^{2}\right)$ & $24.2 \pm 4.0$ & $24.7 \pm 3.4$ & 0.386 \\
\hline Smoking (n (\%)) & $20(20.6)$ & $27(25.5)$ & 0.413 \\
\hline Alcohol drinking (n (\%)) & $18(18.6)$ & $24(22.6)$ & 0.473 \\
\hline Less exercise (n (\%)) & $26(26.8)$ & $26(24.5)$ & 0.711 \\
\hline $\mathrm{MAP}(\mathrm{mmHg})$ & $99.1 \pm 13.8$ & $97.3 \pm 12.6$ & 0.352 \\
\hline Urinary protein $(\mathrm{g} / \mathrm{d})$ & $1.78 \pm 1.80$ & $1.58 \pm 1.32$ & 0.398 \\
\hline Serum albumin $(\mathrm{g} / \mathrm{L})$ & $37.4 \pm 6.0$ & $39.6 \pm 5.5$ & 0.009 \\
\hline eGFR (ml/min) & $80.2 \pm 29.0$ & $80.3 \pm 28.7$ & 0.985 \\
\hline Serum uric acid $(\mu \mathrm{mol} / \mathrm{L})$ & $374.2 \pm 97.6$ & $389.7 \pm 103.7$ & 0.275 \\
\hline CKD stage (n (\%)) & & & 0.989 \\
\hline stage 1 & $43(44.3)$ & $45(42.5)$ & \\
\hline stage 2 & $26(26.8)$ & $29(27.4)$ & \\
\hline stage 3 & $25(25.8)$ & $28(26.4)$ & \\
\hline stage 4 & $3(3.1)$ & $4(3.8)$ & \\
\hline Treatment $^{*}(\mathrm{n}(\%))$ & $45(46.4)$ & $58(54.7)$ & 0.236 \\
\hline Follow-up (months) & $64.6 \pm 17.8$ & $63.4 \pm 14.5$ & 0.600 \\
\hline Endpoint event (n (\%)) & $23(23.7)$ & $9(8.5)$ & 0.003 \\
\hline
\end{tabular}

*Corticosteroid and/or immunosuppressant treatments; BMI: body mass index; MAP: mean arterial pressure; eGFR: estimated glomerular filtration rate; CKD: chronic kidney disease; endpoint event: 50\% decline in eGFR or end-stage renal disease. 
TABLE 4: Clinical characteristics at biopsy and follow-up for patients with blood-stasis BC and non-blood-stasis BC.

\begin{tabular}{|c|c|c|c|}
\hline Characteristics & Blood-stasis BC $(n=64)$ & Non- Blood-stasis BC $(n=139)$ & $P$ values \\
\hline Age (years) & $39.6 \pm 10.4$ & $40.3 \pm 12.1$ & 0.689 \\
\hline Male (n (\%)) & $19(29.7)$ & $92(66.2)$ & 0.001 \\
\hline $\operatorname{BMI}\left(\mathrm{kg} / \mathrm{m}^{2}\right)$ & $24.5 \pm 4.3$ & $24.4 \pm 3.4$ & 0.955 \\
\hline Smoking (n (\%)) & $12(18.8)$ & $35(25.2)$ & 0.313 \\
\hline Alcohol drinking (n (\%)) & $14(21.9)$ & $28(20.1)$ & 0.777 \\
\hline Less exercise (n (\%)) & $14(21.9)$ & $38(27.3)$ & 0.407 \\
\hline $\mathrm{MAP}(\mathrm{mmHg})$ & $98.5 \pm 13.1$ & $98.0 \pm 13.3$ & 0.819 \\
\hline Urinary protein $(\mathrm{g} / \mathrm{d})$ & $1.92 \pm 1.70$ & $1.56 \pm 1.49$ & 0.144 \\
\hline Serum albumin $(\mathrm{g} / \mathrm{L})$ & $37.0 \pm 5.8$ & $39.4 \pm 5.7$ & 0.008 \\
\hline eGFR (ml/min) & $77.1 \pm 30.0$ & $81.7 \pm 28.2$ & 0.299 \\
\hline Serum uric acid $(\mu \mathrm{mol} / \mathrm{L})$ & $410.5 \pm 118.7$ & $369.3 \pm 89.0$ & 0.007 \\
\hline CKD stage (n (\%)) & & & 0.739 \\
\hline stage 1 & $25(39.1)$ & $63(45.3)$ & \\
\hline stage 2 & $17(26.6)$ & $38(27.3)$ & \\
\hline stage 3 & $19(29.7)$ & $34(24.5)$ & \\
\hline stage 4 & $3(4.7)$ & $4(2.9)$ & \\
\hline Treatment $^{*}(\mathrm{n}(\%))$ & $31(48.4)$ & $72(51.8)$ & 0.656 \\
\hline Follow-up (months) & $64.8 \pm 17.3$ & $63.6 \pm 15.6$ & 0.624 \\
\hline Endpoint event (n (\%)) & $18(28.1)$ & $14(10.1)$ & 0.001 \\
\hline
\end{tabular}

* Corticosteroid and/or immunosuppressant treatments; BMI: body mass index; MAP: mean arterial pressure; eGFR: estimated glomerular filtration rate; CKD: chronic kidney disease; endpoint event: 50\% decline in eGFR or end-stage renal disease.

TABLE 5: Factors influencing renal survival from endpoint event by univariate and multivariate Cox regression.

\begin{tabular}{|c|c|c|c|c|}
\hline \multirow{2}{*}{ Characteristics } & \multicolumn{2}{|c|}{ Univariate } & \multicolumn{2}{|c|}{ Multivariate } \\
\hline & $\mathrm{HR}(95 \% \mathrm{CI})$ & $P$ value & $\mathrm{HR}(95 \% \mathrm{CI})$ & $P$ value \\
\hline Hypertension & $6.5(2.9-14.5)$ & $<0.001$ & $3.5(1.4-8.7)$ & 0.009 \\
\hline Renal impairment & $10.6(4.4-25.8)$ & $<0.001$ & $5.8(2.2-15.4)$ & $<0.001$ \\
\hline Yang-deficiency BC & $2.3(1.1-5.1)$ & 0.033 & $2.3(1.0-5.2)$ & 0.041 \\
\hline Blood-stasis BC & $2.3(1.2-4.8)$ & 0.019 & $2.5(1.2-5.2)$ & 0.017 \\
\hline Proteinuria of more than $1 \mathrm{~g} / \mathrm{d}$ & $2.4(1.0-5.4)$ & 0.037 & & NS \\
\hline Hypoproteinemia & $2.8(1.3-5.8)$ & 0.007 & & NS \\
\hline Hyperuricemia & $7.3(3.3-15.8)$ & $<0.001$ & & NS \\
\hline
\end{tabular}

Hypertension: blood pressure $>140 / 90 \mathrm{mmHg}$ or requirement for antihypertensive therapy; renal impairment: eGFR $<60 \mathrm{~mL} / \mathrm{min}$. Hypoproteinemia: serum albumin $<35 \mathrm{~g} / \mathrm{L}$; hyperuricemia: serum uric acid $>420 \mu \mathrm{mol} / \mathrm{L} ;$ NS: not significant.

was associated with worse renal survival from renal endpoint event (log-rank, $4.84 ; P=0.028)$.

3.3. Distribution of Blood-Stasis BC. The patients were divided into blood-stasis BC group $(64,31.5 \%)$ and nonblood-stasis BC group $(139,68.5 \%)$. At baseline, there were no differences between the two groups in clinical characteristics such as age, BMI, lifestyle (smoking, alcohol drinking, and less exercise), MAP, initial proteinuria, eGFR, CKD stage, and corticosteroid and/or immunosuppressant treatment. The male percentage was lower in the blood-stasis BC group than the non-blood-stasis $\mathrm{BC}$ group $(P=0.001)$. The serum albumin level was lower in the blood-stasis $\mathrm{BC}$ group than the non-blood-stasis BC group $(P=0.008)$. The serum uric acid level was higher in the blood-stasis BC group than the nonblood-stasis BC group $(P=0.007)$. During follow-up, more patients achieved the renal endpoint in blood-stasis BC group
(18 (28.1\%)) than non-blood-stasis BC group (14(10.1\%), $P=0.001$, Table 4). Blood-stasis BC was associated with worse renal survival from renal endpoint event (log-rank, 5.96; $P=0.015)$.

3.4. Risk Factors. Univariate analysis was used to indicate factors associated with an increased risk for the endpoint event $(P<0.05)$. These included initial proteinuria of more than $1 \mathrm{~g} / \mathrm{d}$, hypertension, renal impairment $(\mathrm{eGFR}<60 \mathrm{~mL} / \mathrm{min})$, hypoproteinemia (serum albumin $<35 \mathrm{~g} / \mathrm{L}$ ), hyperuricemia (serum uric acid $>420 \mu \mathrm{mol} / \mathrm{L}$ ), Yang-deficiency BC, and blood-stasis BC at the time of renal biopsy. Sex, age, BMI, smoking, alcohol drinking, less exercise, and other seven $\mathrm{BC}$ types were not risk factors for disease progression (Table 5). These factors significantly associated with disease progression by univariate analysis were further analyzed in multivariate Cox regression. It identified that only hypertension 
(hazard ratios (HR) 3.5, $P=0.009$ ), renal impairment (HR 5.8, $P<0.001$ ), Yang-deficiency BC (HR 2.3, $P=0.041$ ), and bloodstasis BC (HR 2.5, $P=0.017$ ) at biopsy were independent predictors of unfavorable renal outcomes (Table 5).

\section{Discussion}

The BC, appearing first in "Huang Di Nei Jing" (a classics textbook of TCM), is one of the most important characteristics of complementary and alternative medicine [5]. BC is composed of physiologic functions and psychological states [12]. The BC theory has been used to guide disease prevention, healthcare, and medical practice by TCM practitioners for more than 2000 years [13]. Patients with the same disease can be treated differently according to their BC types, which is known as "tong bing yi zhi" in TCM. In addition, the susceptibility and progression of diseases will be different in people with different BC types $[2,14]$.

In this study, $98.0 \%$ of IgA nephropathy patients could be classified for at least one $\mathrm{BC}$ type, it confirmed that the CCMQ was suitable for BC identification in IgA nephropathy patients. This study found that the distribution of BC was different between IgA nephropathy patients and normal population; it indicated that $\mathrm{BC}$ was associated with the susceptibility of IgA nephropathy; this is consistent with TCM theory. Same as previous study in Hong Kong Chinese populations [15], we found that the most common BC type was Qi-deficiency BC type in IgA nephropathy patients. This study showed that $67.5 \%$ of IgA nephropathy patients had more than one imbalanced $\mathrm{BC}$ type; overlapping $\mathrm{BC}$ types were very common in IgA nephropathy, which showed the implication for TCM individualized treatment.

Same as previous report $[4,16]$, this study confirmed that male patients were less likely to be Yang-deficiency BC or blood-stasis BC in IgA nephropathy; this is consistent with TCM theory. In the "Yin-Yang" theory of TCM, the male is characterized by "Yang", while the female is characterized by "Yin". This study found that the serum albumin level was lower in the Yang-deficiency BC group than the nonYang-deficiency BC group; this difference was also found in patients with and without blood-stasis BC. In recent years, some studies had indicated the molecular mechanisms and polymorphisms of Yang-deficient BC and blood-stasis BC $[16,17]$. Maybe, there were some kinds of corresponding relations between these $\mathrm{BC}$ types and the decrease in expression level of certain proteins and require more research. This study showed that the serum uric acid level was higher in the bloodstasis BC group than the non-blood-stasis BC group and require more research.

IgA nephropathy, characterized by IgA deposition in the glomerular mesangium, is not a benign disease [11]. In this study, although many patients were biopsied at a relatively early stage with $70.4 \%$ of patients in CKD stages 1 and 2 and none in stage 5, during follow-up, $15.8 \%$ of patients achieved the renal endpoint. So, it is necessary to pay more attentions to the risk factors for the progression of IgA nephropathy.

To the best of our knowledge, this is the first report showing that Yang-deficient BC and blood-stasis BC may be independent predictors for the progression of IgA nephropathy. And these new predictive factors are noninvasive and without incurring any costs for BC identification. In TCM, the constitution, inherently forming and developing throughout life, is relatively stable. On the other hand, the constitution is changeable; with the course of diseases lasting, the participants' constitution statuses might change. We will evaluate the participants' constitution types again in the future. In TCM, the constitution is adjustable; therefore, the treatment of tonifying Yang and promoting blood circulation and removing blood-stasis might improve the prognosis for IgA nephropathy patients. Consistent with the previous studies [11, 18], in our study, hypertension was a strong risk factor for the progression of IgA nephropathy. Nevertheless, blood pressure control in China is unsatisfactory; rigorous blood pressure control might improve the prognosis greatly for IgA nephropathy patients [11].

In multivariate analysis, initial proteinuria was not associated with renal outcomes of IgA nephropathy. This finding is contrary to previous reports [18], but similar to Lv's study [11]. In this study, most of our patients had severe proteinuria; $53.7 \%$ showed proteinuria greater than $1.0 \mathrm{~g} / \mathrm{d}$; $11.3 \%$ with proteinuria greater than $3.5 \mathrm{~g} / \mathrm{d} .50 .7 \%$ of the patients accepted corticosteroid and/or immunosuppressant treatment. Previous report found that patients with mild renal lesions were easy to rapidly enter proteinuria remission [11]. Proteinuria level and remission or not may be partly responsible for these differences. In addition, measuring the time-average proteinuria during follow-up may be more accurate than point proteinuria in baseline for judging the prognosis of IgA nephropathy [18]. A few studies showed that sex, age, BMI at biopsy were also associated with the progression of IgA nephropathy $[9,19,20]$; inconsistent with these previous studies, no such associations were found in our study.

In this study, the histological lesions were not analyzed, for our previous study aimed at renal lesions and found that intrarenal arterial lesions were association with the prognosis of IgA nephropathy [21]. In addition, the limitations of this study included recruitment of participants from a single medical center and lack of BC status evaluating in followup.

\section{Conclusions}

Most patients of IgA nephropathy were biopsied at an early stage. Yang-deficiency BC and blood-stasis BC at biopsy were most closely associated with the worse prognosis of $\operatorname{IgA}$ nephropathy along with hypertension and renal impairment. Further studies on the role of BC in progression of $\operatorname{IgA}$ nephropathy are warranted.

\section{Data Availability}

The data used to support the findings of this study are available from the corresponding author upon request.

\section{Conflicts of Interest}

The authors declare that there are no conflicts of interest. 


\section{Acknowledgments}

This study was supported by the National Natural Science Foundation of China (no. 81500566) and the Medicine and Health Science and Technology Projects of Zhejiang Province (no. 2018KY603). The authors would like to thank all subjects for their participation in this study. The authors appreciate Professor Xueguang Zhang and Professor Jie Wu for diagnosing of IgA nephropathy. The authors also thank Xiaorui Liu and Liwei Zhang for patients recruiting and following.

\section{References}

[1] J. S. Lin, L. L. Chen, J. D. Lin et al., "Bcqs: a body constitution questionnaire to assess Yin-Xu. Part II: evaluation of reliability and validity," Forsch Komplementmed, vol. 19, no. 6, pp. 285-292, 2012.

[2] J. Wang, Q. Wang, L. Li et al., "Phlegm-dampness constitution: genomics, susceptibility, adjustment and treatment with traditional Chinese medicine," American Journal of Chinese Medicine, vol. 41, no. 2, pp. 253-262, 2013.

[3] X. Liu, L. Liu, P. Chen et al., "Clinical trials of traditional Chinese medicine in the treatment of diabetic nephropathy-a systematic review based on a subgroup analysis," Journal of Ethnopharmacology, vol. 151, no. 2, pp. 810-819, 2014.

[4] Y. Cen, R. K.-K. Leung, F. Zhang et al., "The association between Yang-Deficient Constitution and clinical outcome of highly active antiretroviral therapy on people living with HIV," Evidence-Based Complementary and Alternative Medicine, vol. 2013, Article ID 201857, 9 pages, 2013.

[5] K.-C. Huang, C.-C. Chen, Y.-C. Su et al., "The relationship between stasis-stagnation constitution and peripheral arterial disease in patients with type 2 diabetes," Evidence-Based Complementary and Alternative Medicine, vol. 2014, Article ID 903798, 6 pages, 2014.

[6] G.-Y. Cai and X.-M. Chen, "Immunoglobulin a nephropathy in China: Progress and challenges," American Journal of Nephrology, vol. 30, no. 3, pp. 268-273, 2009.

[7] P.-C. Shen, L.-Q. He, Y. Tang, Q. Wang, W. Wang, and J. $\mathrm{Li}$, "Clinicopathological characteristics and prognostic factors of asymptomatic IgA nephropathy," Journal of Investigative Medicine, vol. 58, no. 3, pp. 560-565, 2010.

[8] L. Tian, X. Shao, Y. Xie et al., "The long-term efficacy and safety of immunosuppressive therapy on the progression of IgA nephropathy: A meta-analysis of controlled clinical trials with more than 5-year follow-up," Expert Opinion on Pharmacotherapy, vol. 16, no. 8, pp. 1137-1147, 2015.

[9] G.-Y. Cheng, D.-W. Liu, N. Zhang, L. Tang, Z.-Z. Zhao, and Z.S. Liu, "Clinical and prognostic implications of serum uric acid levels on IgA nephropathy: A cohort study of 348 cases with a mean 5-year follow-up," Clinical Nephrology, vol. 80, no. 1, pp. 40-46, 2013.

[10] L. A. Stevens, M. A. Claybon, C. H. Schmid et al., "Evaluation of the chronic kidney disease epidemiology collaboration equation for estimating the glomerular filtration rate in multiple ethnicities," Kidney International, vol. 79, no. 5, pp. 555-562, 2011.

[11] J. Lv, H. Zhang, Y. Zhou, G. Li, W. Zou, and H. Wang, "Natural history of immunoglobulin A nephropathy and predictive factors of prognosis: A long-term follow up of 204 cases in China," Nephrology, vol. 13, no. 3, pp. 242-246, 2008.
[12] Q. Wang, "Individualized medicine, health medicine, and constitutional theory in Chinese medicine," Frontiers of Medicine, vol. 6, no. 1, pp. 1-7, 2012.

[13] J. Wang, Y. Li, C. Ni, H. Zhang, L. Li, and Q. Wang, "Cognition research and constitutional classification in Chinese medicine," American Journal of Chinese Medicine, vol. 39, no. 4, pp. 651660, 2011.

[14] Q. Wang, X.-J. Ren, S.-L. Yao, and H.-D. Wu, "Clinical observation on the endocrinal and immune functions in subjects with yin-deficiency constitution," Chinese Journal of Integrative Medicine, vol. 16, no. 1, pp. 28-32, 2010.

[15] W. Wong, C. L. K. Lam, V. T. Wong et al., "Validation of the constitution in Chinese medicine questionnaire: does the traditional chinese medicine concept of body constitution exist?" Evidence-Based Complementary and Alternative Medicine, vol. 2013, Article ID 481491, 14 pages, 2013.

[16] K.-C. Huang, H.-J. Huang, C.-C. Chen et al., "Susceptible gene of stasis-stagnation constitution from genome-wide association study related to cardiovascular disturbance and possible regulated traditional Chinese medicine," BMC Complementary and Alternative Medicine, vol. 15, no. 1, p. 229, 2015.

[17] Y. Wu, Y. Cun, J. Dong et al., "Polymorphisms in PPARD, PPARG and APM1 associated with four types of Traditional Chinese Medicine constitutions," Journal of Genetics and Genomics, vol. 37, no. 6, pp. 371-379, 2010.

[18] W. B. Le, S. S. Liang, Y. L. Hu et al., "Long-term renal survival and related risk factors in patients with IgA nephropathy: Results from a cohort of 1155 cases in a Chinese adult population," Nephrology Dialysis Transplantation, vol. 27, no. 4, pp. 1479-1485, 2012.

[19] S. Kato, A. Nazneen, Y. Nakashima et al., "Pathological influence of obesity on renal structural changes in chronic kidney disease," Clinical and Experimental Nephrology, vol. 13, no. 4, pp. 332-340, 2009.

[20] M. Goto, K. Wakai, T. Kawamura, M. Ando, M. Endoh, and Y. Tomino, "A scoring system to predict renal outcome in IgA nephropathy: A nationwide 10-year prospective cohort study," Nephrology Dialysis Transplantation, vol. 24, no. 10, pp. 30683074, 2009.

[21] S. W. Duan, S. W. Liu, X. F. Sun et al., "Potential association of hyperhomocysteinemia with the progression of IgA nephropathy: A retrospective study," Chinese Medical Journal, vol. 127, no. 10, pp. 1849-1852, 2014. 


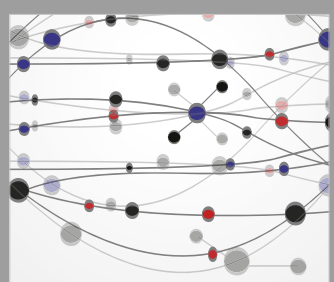

The Scientific World Journal
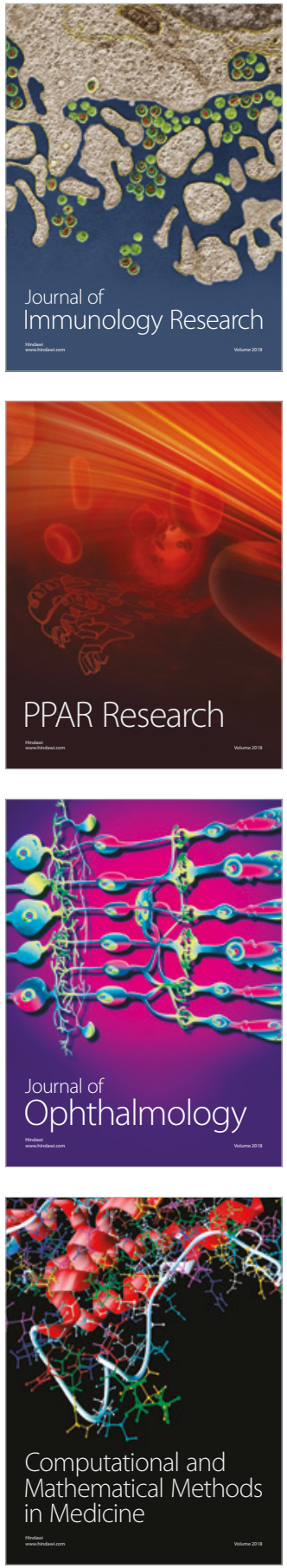

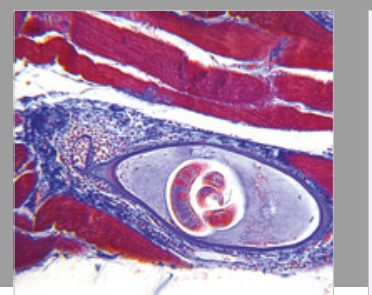

Gastroenterology Research and Practice

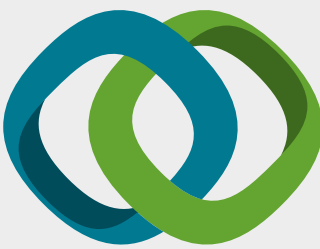

\section{Hindawi}

Submit your manuscripts at

www.hindawi.com
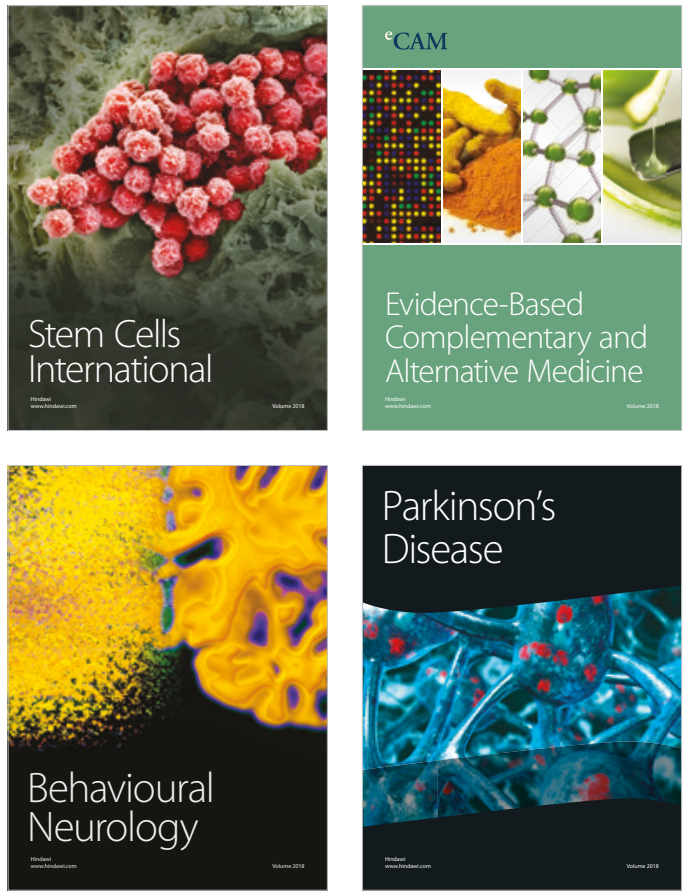

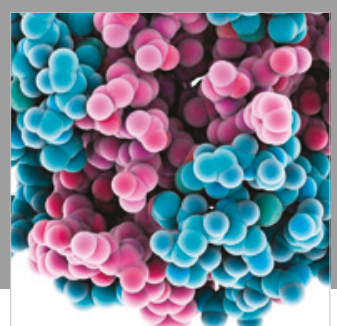

ournal of

Diabetes Research

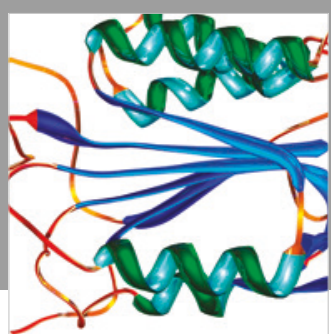

Disease Markers
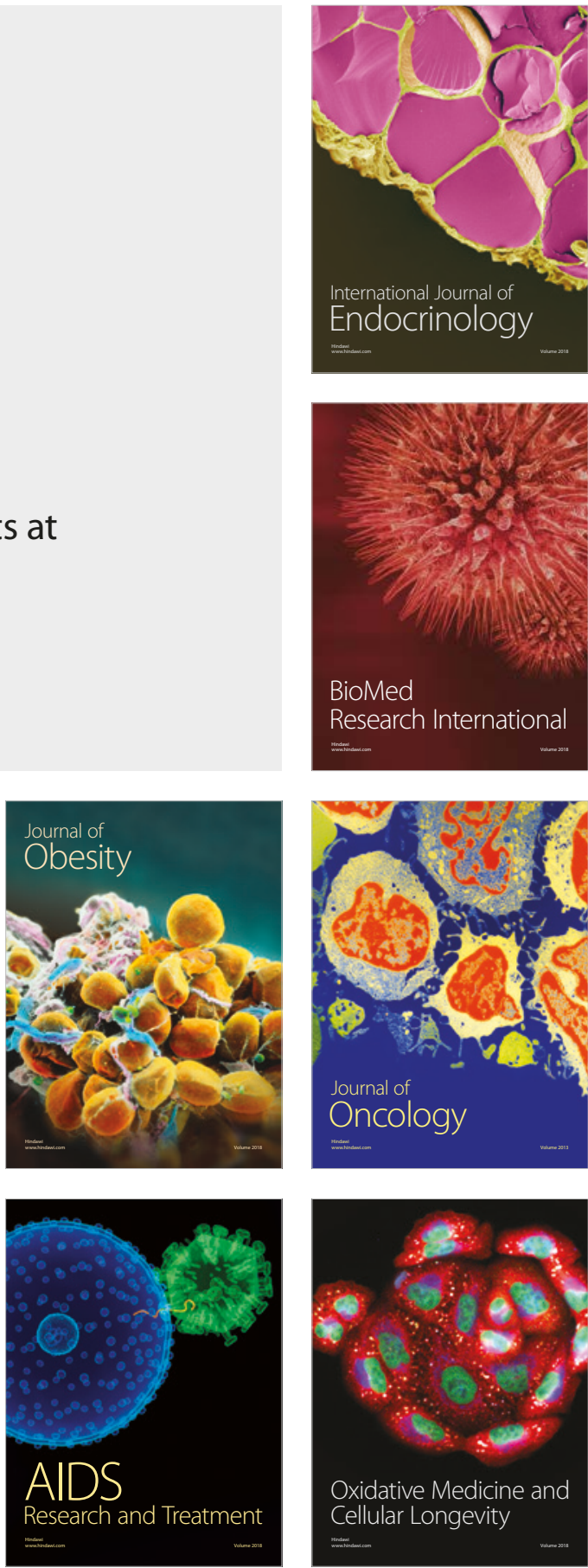